\title{
Facciamogliela pagare
}

\section{Let them pay for it}

Giovanni Lodi

Dipartimento di Scienze Biomediche, Chirurgiche e Odontoiatriche

Università degli Studi di Milano

via Beldiletto 1/3 Milano 20142

Italia

giovanni.lodi@unimi.it

tel. + 390250319021

fax. +390250319041

"E' la nostra suprema potenza, non soltanto perché è lo snodo fondamentale per risolvere i problemi dei malati, ma anche perché è l'atto intellettualmente più nobile della nostra professione." (V. Cagli 2007)

La citazione, per chi non lo avesse intuito, si riferisce alla diagnosi che fin dalle prime lezioni all'università ci viene insegnato essere l'attività che distingue e definisce il medico rispetto alle altre figure professionali che si occupano di salute. Le quali possono somministrare terapie o prestare trattamenti, ma non fare diagnosi. Come certificato dal codice deontologico della nostra professione, dove si afferma che "la diagnosi a fini preventivi, terapeutici, e riabilitativi, è una diretta, esclusiva e non delegabile competenza del medico". Per dire, la diagnosi di gengivite (!) può essere fatta solo dall'odontoiatra, anche se poi potrà essere l'igienista a risolvere il problema grazie a un'accurata terapia causale.

Difficile mettere in discussione questi principi. Però a me sorge più di un dubbio, almeno per quanto riguarda la nostra professione. Non sulla base di un qualche impianto teorico, ma per un dato di realtà che è sotto gli occhi di tutti.

Infatti, se nelle pubblicità di catene odontoiatriche grandi e piccole la prima visita è invariabilmente gratuita (e chissà se mettere i due terzi del capitale sociale in mano a odontoiatri cambierebbe la cosa), se i pazienti sono convinti che una nostra visita sia non fare niente e i colleghi con cui affronto l'argomento mi obiettano che è difficile chiedere una parcella quando non si è fatto niente, se comunque tutti fanno così, se per molti pazienti (e non solo) i momenti della visita diagnostica e della stesura del piano di cure sono sintetizzati nella preparazione del preventivo, se sono una minoranza gli odontoiatri che rilasciano una relazione scritta al termine della prima visita, se tutto questo è vero, ed è vero, allora quella sulla diagnosi è solo retorica, buona per prefazioni di libri, prolusioni di congressi e discorsi ai neolaureati.

Non è una questione di soldi, credo sia chiaro, ma di salvaguardia del nostro essere medici.

Buona lettura 